\title{
VLBA Observations of Radio Reference Frame Sources. II. Astrometric Suitability Based on Observed Structure
}

\author{
A. L. Fey \\ United States Naval Observatory, Washington, D.C. \\ P. Charlot \\ Paris Observatory, Paris, France
}

\begin{abstract}
We use source models derived from VLBA observations of 169 extragalactic sources to quantify the expected effects of intrinsic source structure on astrometric bandwidth synthesis VLBI observations. We define a source "structure index" based on the median structure corrections and suggest that this index can be used as an estimate of the astrometric quality of the sources. A correlation between the compactness of the sources and their formal position uncertainties was found indicating that the more extended sources have larger formal position uncertainties.
\end{abstract}

\section{Introduction}

Many of the most intense compact extragalactic radio sources are used routinely to determine tectonic plate motion to accuracies on the order of several millimeters per year, to determine Earth rotation parameters to accuracies on the order of $0.1 \mathrm{mas}$, and to strengthen the terrestrial reference frame. Extragalactic radio sources are assumed to be very distant and thus should exhibit little or no detectable proper motions. However, temporal variations of the intrinsic structure of these sources will result in apparent motion when astrometric observations are made at several epochs. In order to quantify this effect, we have started an observing program to image selected extragalactic sources on a regular basis. Initial results of these observations are reported by Fey, Clegg, \& Fomalont (1996) and by Fey \& Charlot (1997).

\section{Source Structure Corrections and the Structure Index}

The method used to calculate source structure corrections to the VLBI delay observable is described by Charlot (1990). We use their analysis to obtain an estimate of the overall magnitude of intrinsic structure effects on a hypothetical bandwidth synthesis delay measurement. The structure delay is calculated for all pixels of a $512 \times 512(u, v)$ grid lying within a circle of radius equal to the diameter of the Earth. CLEAN component models are used as estimates of the intrinsic source structure. The mean, rms, maximum, and median values of the structure corrections (absolute values) are determined and the calculation is done separately for each observation frequency ( 2 and $8 \mathrm{GHz}$ ). Results of this analysis are reported by Fey \& Charlot (1997) and show that intrinsic structure contributions to the measured delay are significant, ranging from maximum delay corrections at $8 \mathrm{GHz}$ of only a few picoseconds for the most compact sources to maximum corrections of several nanoseconds for the most extended sources.

Next, the sources are grouped into four classes based on the median value of the calculated structure delay corrections, $\tau_{\text {median }}$. The "structure index," SI is defined as follows: $\mathrm{SI}=1,0 \mathrm{ps} \leq \tau_{\text {median }}<3 \mathrm{ps}$; $\mathrm{SI}=2,3 \mathrm{ps} \leq \tau_{\text {median }}<10 \mathrm{ps}$; 
$\mathrm{SI}=3,10 \mathrm{ps} \leq \tau_{\text {median }}<30 \mathrm{ps} ; \mathrm{SI}=4,30 \mathrm{ps} \leq \tau_{\text {median }}<\infty$. The structure index can be used as an estimate of the astrometric quality of the observed sources.

\section{Results}

Table 1 shows a comparison between the $8 \mathrm{GHz}$ structure index of the observed sources and the formal uncertainties of their astrometric positions obtained from the catalog of Johnston et al. (1995). Results of this comparison indicate that, in the mean, sources with larger position formal uncertainties have a larger structure index than those with smaller uncertainties, as would be expected if the structure index accurately traces the complexity of intrinsic source structure.

Table 1. Structure Index Versus Formal Position Uncertainty

\begin{tabular}{ccccc}
\hline \hline $\begin{array}{c}\text { Structure } \\
\text { Index }\end{array}$ & $\begin{array}{c}\text { Number of } \\
\text { Sources }\end{array}$ & $\begin{array}{c}\text { Mean } \\
N_{\text {obs }}\end{array}$ & $\begin{array}{c}\sigma_{\alpha} \cos (\delta) \\
\text { (mas) }\end{array}$ & $\begin{array}{c}\text { Mean Uncertainty } \\
\text { (mas) }\end{array}$ \\
\hline 1 & 29 & $2637 \pm 1128$ & $0.15 \pm 0.03$ & $0.19 \pm 0.03$ \\
2 & 70 & $6381 \pm 1727$ & $0.22 \pm 0.04$ & $0.29 \pm 0.06$ \\
3 & 46 & $3540 \pm 1125$ & $0.34 \pm 0.12$ & $0.46 \pm 0.15$ \\
4 & 23 & $683 \pm 410$ & $1.50 \pm 0.76$ & $1.03 \pm 0.37$ \\
\hline
\end{tabular}

Table 2 shows a comparison between the $8 \mathrm{GHz}$ structure index and the 8 $\mathrm{GHz}$ core flux density of the observed sources. Since the core to total flux density ratio is an indication of the "compactness" of a source, results of this comparison indicate that, in the mean, more extended sources have a larger structure index than more compact sources. Thus, the compactness of the sources has a direct bearing on the structure index.

Table 2. Structure Index Versus Core Flux Density

\begin{tabular}{ccc}
\hline $\begin{array}{c}\text { Structure } \\
\text { Index }\end{array}$ & $\begin{array}{c}\text { Number of } \\
\text { Sources }\end{array}$ & $\begin{array}{c}\text { Mean Ratio } \\
S_{\text {core }} / S_{\text {total }}\end{array}$ \\
\hline 1 & 29 & $0.92 \pm 0.01$ \\
2 & 71 & $0.82 \pm 0.01$ \\
3 & 44 & $0.64 \pm 0.02$ \\
4 & 22 & $0.48 \pm 0.04$ \\
\hline
\end{tabular}

Comparison of Table 1 with Table 2 then indicates that the more extended sources have larger formal position uncertainties.

\section{References}

Charlot, P. 1990. AJ, 99, 1309-1326.

Fey, A. L., Clegg, A. W., \& Fomalont, E. B. 1996. ApJS, 105, 299-330.

Fey, A. L., \& Charlot, P. 1997. ApJS, 111, 95-142.

Johnston, K. J., et al. 1995. $A J, 110,880-915$. 